\title{
The association of lymphotoxin-beta receptor with the subsequent diagnosis of incident gastrointestinal cancer: results from the Dallas Heart Study
}

\author{
Colin P. Bergstrom ${ }^{1}$, Muhammad S. Beg ${ }^{2}$, Colby Ayers ${ }^{3}$, Arjun Gupta ${ }^{1}$, Ian J. Neeland ${ }^{4}$ \\ ${ }^{1}$ Department of Internal Medicine, ${ }^{2}$ Division of Oncology, ${ }^{3}$ Department of Clinical Sciences, ${ }^{4}$ Division of Cardiology, University of Texas \\ Southwestern Medical Center, Dallas, TX, USA \\ Contributions: (I) Conception and design: CP Bergstrom, MS Beg, IJ Neeland; (II) Administrative support: MS Beg, IJ Neeland; (III) Provision of \\ study materials or patients: CP Bergstrom, MS Beg, IJ Neeland; (IV) Collection and assembly of data: CP Bergstrom, MS Beg, C Ayers, IJ Neeland; (V) \\ Data analysis and interpretation: All authors; (VI) Manuscript writing: All authors; (VII) Final approval of manuscript: All authors. \\ Correspondence to: Colin P. Bergstrom, MD. Department of Internal Medicine, University of Texas Southwestern Medical Center, 5323 Harry Hines \\ Boulevard, Dallas, TX 75390-8852, USA. Email: Colin.Bergstrom@phhs.org.
}

\begin{abstract}
Background: Lymphotoxin-beta receptor (LT $\beta$ R) is an immunological protein associated with inflammation, and from preclinical studies is implicated in tumorigenesis. The epidemiological relationships with cancer are unknown, hence this study investigated their associations.

Methods: From a multiethnic population-based cohort, 3,032 participants without a prevalent cancer (a diagnosis prior to or within one year of enrollment) at baseline underwent measurement of plasma LT $\beta$ R. These participants were followed for incident cancer using the Texas Cancer Registry (TCR).

Results: Over a median follow-up of 12.1 years, 178 participants developed incident cancer, of which 30 participants developed incident gastrointestinal (GI) cancer. Median plasma LT $\beta R$ (1.10 vs. $1.00 \mathrm{ng} / \mathrm{mL}$, $\mathrm{P}<0.02)$ levels were higher in individuals with overall incident cancer compared to those without cancer. After adjustments for age, sex, and race/ethnicity, these relationships were no longer significant. When analyses were stratified by cancer type, LT $\beta$ R was positively associated with GI cancer after adjustments: HR, 95\% CI per 1-standard deviation increase in concentration 2.64 (1.23-5.68), $\mathrm{P}=0.013$. LT $\beta \mathrm{R}$ stratified by quartiles was significantly associated temporally with the risk of incident GI cancer, log-rank: $\mathrm{P}=0.011$. The median interval to incident GI cancer diagnosis was 5.9 years.

Conclusions: Increased plasma levels of LT $\beta R$ are associated with the development of GI cancer. The antecedent findings years prior to a subsequent diagnosis of incident GI cancer suggest a role for LT $\beta \mathrm{R}$ in the pathogenesis of GI cancer. Further studies are needed to determine if LT $\beta$ R can serve as an immune biomarker for GI cancer, in particular hepatocellular and colorectal cancers.
\end{abstract}

Keywords: Inflammation; immunology; gastrointestinal cancer (GI cancer); hepatocellular carcinoma; colorectal carcinoma; epidemiology

Submitted Oct 29, 2019. Accepted for publication Dec 06, 2019.

doi: 10.21037/jgo.2020.01.04

View this article at: http://dx.doi.org/10.21037/jgo.2020.01.04

\section{Introduction}

Inflammation is considered an enabling characteristic for cancer development and tumorigenesis (1). The link is particularly strong between tumorigenesis and chronic gastrointestinal (GI) inflammatory conditions
$(2,3)$. Moreover, inflammatory cells and mediators of the immune system are commonly detected in GI cancers (4). This link has been reported in epidemiological studies which have demonstrated that circulating mediators of the inflammatory and immune responses are associated with the 
development of GI cancer (5-8). Other studies have failed to demonstrate the association of circulating mediators of the inflammatory and immune responses with GI cancer (9).

Lymphotoxin- $\beta$ receptor (LT $\beta R$ ) is a protein that mediates various immunological and inflammatory pathways (10-12). LT $\beta R$ is a cell surface receptor and a member of the tumor necrosis factor receptor superfamily (TNFRSF3) expressed on a variety of cells such as epithelial, fibroblasts, endothelial and myeloid lineage cells but not B or T lymphocytes (13). Activation of LT $\beta \mathrm{R}$ allows for communication between lymphocytes and stromal, epithelial or myeloid cells, influencing immunological and inflammatory pathways $(10-12,14)$. Two recent independent reviews of a host of preclinical animal studies separately concluded that LT $\beta R$ promotes tumorigenesis $(15,16)$. An essential mechanistic role for LT $\beta \mathrm{R}$ in the tumorigenesis of hepatocellular carcinoma was demonstrated by the provocative studies of Haybaeck and colleagues (17). Moreover, lymphotoxin has been implicated in shaping the network pathways of the tumor microenvironment, which conceptually is an early event in tumorigenesis (10).

Epidemiological studies have investigated the associations of inflammatory biomarkers with cardiovascular disease (18-23) and concurrently, within these same cohorts, investigations have explored the association of inflammatory biomarkers with incident cancer (6-8). Given the scientific evidence that LT $\beta \mathrm{R}$ is associated with early tumorigenesis for GI cancers, we aimed to investigate the relationship of this inflammatory protein with the subsequent development of incident cancer and incident GI cancer, leveraging an existing, large, and multiethnic population-based cohort: the Dallas Heart Study (DHS) to explore this hypothesis.

\section{Methods}

\section{Study population}

Details of the design and enrollment of the DHS have been previously described (24). Briefly, the DHS is a well characterized single site, multiethnic, populationbased probability sample of Dallas County residents (aged 18-65 years) which enrolled participants between July 2000 and January 2002. This existing cohort was designed to intentionally oversample non-Hispanic African American participants to compose half of the study population included the collection of detailed socioeconomic, biomarker and imaging data from each participant.

The current study includes participants who completed a detailed computer-assisted survey, measurements of anthropometric variables, biomarker, radiographic imaging, blood pressure and laboratory testing $(\mathrm{n}=3,557)$. Demographic, lifestyle and other risk factors were determined from the baseline questionnaire. Ethnicity was self-assigned in accordance with United States census categories. Body mass index (BMI) was calculated as weight (kilograms) divided by the square of the height (meters). Hypertension was defined as blood pressure $\geq 140 / 90 \mathrm{~mm} \mathrm{Hg}$ or taking antihypertensive medication(s). Diabetes mellitus was defined as a fasting serum glucose $\geq 126 \mathrm{mg} / \mathrm{dL}$, self-reported diabetes, or taking hypoglycemic medication. Smoking was defined as cigarette use within the previous 30 days and/or a lifetime history of having smoked $\geq 100$ cigarettes. Alcohol use was determined in grams/wk by self-report. Participants without measurement of plasma LT $\beta R$ were excluded from the study population $(\mathrm{n}=368)$. As with other studies investigating incident cancer in the DHS cohort, participants with history of or present diagnosis of malignancy were also excluded (25). To account for cancers that may have been undetected at baseline, new cases of cancer diagnosed within one year after date of enrollment to DHS were excluded from the analysis (blanking period). After these exclusions, 3,032 participants were eligible for follow-up (Figure 1). All participants provided written informed consent to participate in the DHS. The Institutional Review Board of the University of Texas Southwestern Medical Center in Dallas approved the protocol and all research was performed in accordance with relevant institutional guidelines.

\section{Assay for LTBR and other analytes}

Fasting venous blood samples were collected in EDTA collection tubes and maintained at $4{ }^{\circ} \mathrm{C}$ for $<4 \mathrm{~h}$, followed by centrifugation at $4{ }^{\circ} \mathrm{C}$. Aliquots of plasma were frozen at $-80{ }^{\circ} \mathrm{C}$ and shipped to Biosite, Inc. (an Inverness Medical Company, Waltham, MA), where they were thawed for measurement of LT $\beta R$ as previously described (23). A Quantikine based sandwich immunoassay was performed using Biosite's proprietary device platform. Quality control and assurance was maintained by Biosite. The minimal detection limit of the assay was $0.3 \mathrm{ng} / \mathrm{mL}$, and the inter assay and intra assay coefficient of variation within the assay range was $14 \%$. Assay results were transferred to University of Texas Southwestern and merged with the clinical database. Biosite had no access to clinical data at any time. Samples had been thawed once for aliquoting prior to 


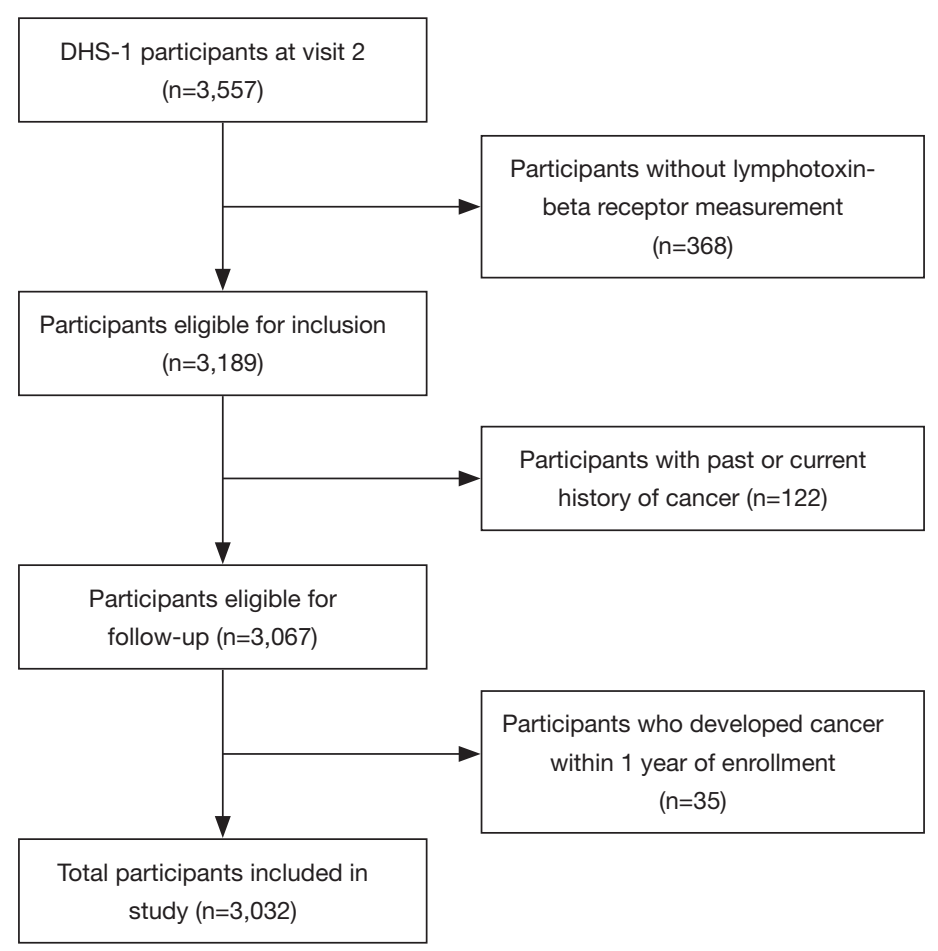

Figure 1 Flow diagram of the Dallas Heart Study for the lymphotoxin- $\beta$ receptor and caspase- 3 study cohort.

LT $\beta$ R measurement. High sensitivity assay for C-reactive protein (CRP) was performed using the Roche/Hitachi 912 System (Roche Diagnostic, Indianapolis, IN) as previously described (24). Assays were performed by individuals blinded to all clinical data.

\section{Cancer assessment}

The utilization of the Texas Cancer Registry (TCR) linked to the DHS cohort for identification and capture of cancer cases has been previously been described $(24,25)$. All cancer cases identified by the TCR were classified by type/location of cancer, date of recorded diagnosis as well as a determination of prevalent or incident cancer. Cases with a date of cancer diagnosis one year after the date of enrollment to DHS were classified as incident cancer and those cases at the time of enrollment or within the oneyear window were classified as prevalent cancer. GI cancers included cancers where the primary site was upper and lower GI tracts as well as pancreatic, liver or gallbladder. Carcinoma in-situ and skin cancers were not included. In cases with more than one known cancer, only the first was included.

\section{Statistical analysis}

Baseline characteristics of the study population are reported as proportions or median values with 25 th and 75 th percentiles (interquartile range, IQR). Univariate comparisons utilized Chi Square tests or Wilcoxon rank sum test, as appropriate. Quartile analysis used a twosided Jonckheere-Terpstra Test to assess trends. KaplanMeier curves were constructed for the relationship of LT $\beta$ R to time of incident cancer cases and assessed by the logrank test. Data for the anatomical sites are expressed as a mean with the standard deviation. Plasma lymphotoxin $\beta$ receptor levels were modeled following a logarithmic transformation. Separate Cox proportional hazards models were used to examine the multivariate adjusted associations between LT $\beta$ R and the primary outcome. The proportionality assumption was verified using Schoenfeld residuals. The results are reported as hazard ratios (HR) and $95 \%$ CI per 1-standard deviation of the biomarker concentration. The primary outcome was any incident cancer and secondary outcomes were incident cancer types (breast, GI and prostate). Models were sequentially adjusted for age, sex, race/ethnicity, smoking history, alcohol history, 
Table 1 Baseline characteristics of participants with and without incident cancer in the Dallas Heart Study

\begin{tabular}{|c|c|c|c|}
\hline Characteristics & No cancer $(n=2,854)$ & Incident cancer $(n=178)$ & P-value \\
\hline Age (years) & 43 [36-51] & 52 [45-58] & $<0.01$ \\
\hline Males & $1,282(44.9)$ & $79(44.4)$ & 0.94 \\
\hline \multicolumn{4}{|l|}{ Race } \\
\hline Caucasians & $832(29.2)$ & $53(29.8)$ & 0.87 \\
\hline Hispanic & $507(17.8)$ & $16(9.0)$ & $<0.01$ \\
\hline Other & $58(2.0)$ & $4(2.2)$ & 0.78 \\
\hline Diabetes & $322(11.3)$ & $38(21.3)$ & $<0.01$ \\
\hline Smoking & $809(28.3)$ & $58(32.6)$ & 0.20 \\
\hline Alcohol use & $1,963(68.8)$ & $118(66.3)$ & 0.56 \\
\hline \multicolumn{4}{|l|}{ Measures of adiposity } \\
\hline Body weight (kg) & $83.0(70.7-98.4)$ & 83.4 (71.6-99.6) & 0.33 \\
\hline Body mass index $\left(\mathrm{kg} / \mathrm{m}^{2}\right)$ & $29.4(25.5-34.6)$ & $30.2(25.1-35.9)$ & 0.30 \\
\hline \multicolumn{4}{|l|}{ Biochemical characteristics } \\
\hline $\begin{array}{l}\text { High sensitivity C-reactive } \\
\text { protein }(\mathrm{mg} / \mathrm{dL})\end{array}$ & $2.8(1.2-6.80)$ & $3.60(1.40-8.00)$ & 0.67 \\
\hline
\end{tabular}

Data reported as median (interquartile range) or number (\%), as appropriate.

family history for cancer, physical activity, BMI and high sensitivity C-reactive protein. Due to the number of events, adjustments of confounders were restricted to major clinical variables such as age, race and sex. Sensitivity analyses were performed by extending the blanking period for incident cancer to two years to exclude potential indolent undetected cancers at baseline. $\mathrm{P}$ values of $<0.05$ were considered statistically significant (23). All analyses were performed using SAS version 9.4 (SAS Corporation, Cary, NC, USA).

\section{Results}

\section{Patient characteristics for incident cancer cohort}

The study cohort of the consisted of 3,032 cancer-free individuals, median age of 43 years [IQR 36, 51]. The cohort was 51.1\% African American, 29.2\% White and $17.8 \%$ Hispanic and $55.1 \%$ female (Table 1) The study population was followed for a median of 12.1 years [IQR $11.7,12.5]$ and 178 (5.9\%) participants were diagnosed with an incident cancer one year after enrollment in the DHS. The most common type of incident cancers was breast, GI and prostate $(22 \%, 17 \%$ and $17 \%$ of the total incident cancer, respectively). Further details regarding type of cancer are presented in Table S1. The participants with incident cancer were older compared to the participants who were cancer free (Table 1). In regards to demographics, for individuals without incident cancer, the majority were women (55.1\%) and African Americans (51.1\%). There was no significant difference in body weight, BMI or measurements of serum high sensitivity $\mathrm{C}$-reactive protein levels between those with and without incident caner. 
Table 2 Associations of plasma lymphotoxin- $\beta$ receptor with incident cancer

\begin{tabular}{lcccc}
\hline & Model 1 & Model 2 & Model 3 & Model 4 \\
\hline Lymphotoxin- $\beta$ receptor & 1.31 (1.06-1.62); 0.014 & $1.10(0.87-1.38) ; 0.44$ & $1.12(0.88-1.43) ; 0.37$ & $1.13(0.88-1.45) ; 0.34$ \\
\hline Data reported as HR (95\% Cl); P value. Model 1, unadjusted (univariable, continuous); Model 2, additionally adjusted for age, race, sex; \\
Model 3, additionally adjusted for age, smoking, alcohol, family history for cancer, physical activity, body mass index; Model 4, additionally \\
adjusted for high sensitivity C-reactive protein levels.
\end{tabular}

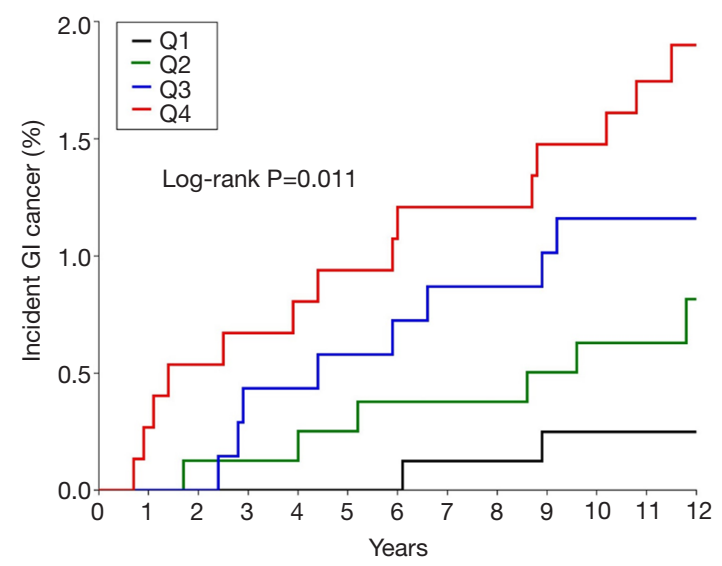

Figure 2 Kaplan Meier curve of time to incident gastrointestinal cancer by lymphotoxin- $\beta$ receptor quartiles. Vertical axis is the percentage of subjects developing incident gastrointestinal cancer in each quartile and the horizontal axis is years of follow-up. (Q1 refers to the first quartile, Q2 refers to the second quartile, Q3 refers to the third quartile and Q4 refers to the fourth quartile). Median interval to GI cancer diagnosis was $5.9(2.8,8.9)$ years. Log-rank test $\mathrm{P}=0.011$.

\section{Plasma LTBR and incident cancer}

Median plasma LT $\beta$ R levels were 1.10 (IQR 0.80, $1.50) \mathrm{ng} / \mathrm{mL}$ in the incident cancer group and 1.00 (IQR 0.70, 1.30) $\mathrm{ng} / \mathrm{mL}$ in the non-cancer group $(\mathrm{P}<0.01)$ (Table 1). Stratification of the study population into quartiles of plasma LT $\beta$ R levels revealed associations with age and female but no association with race/ethnicity (Table S2). In multivariable analysis there also existed an association of LT $\beta R$ levels to age, diabetes, hypertension, alcohol use, and high sensitivity C-reactive protein (Table S3). To further assess the associations of plasma LT $\beta \mathrm{R}$ with incident cancer, four separate models were constructed for plasma LT $\beta R$. In the unadjusted analysis for plasma LT $\beta R$ as depicted in Table 2, LT $\beta$ R was significantly associated with incident cancer with a HR of $1.31(\mathrm{P}=0.014)$. After adjusting for age, race and sex, the HR for incident cancer was attenuated to
$1.10(\mathrm{P}=0.44)$. Additional adjustment for smoking, alcohol, family history of cancer, and for C-reactive protein did not alter the findings.

\section{Plasma LTBR and time to diagnosis for incident GI cancer}

Among the 178 participants with incident cancer, 30 (17\%) were GI. The Kaplan Meier curves for incident GI cancer by quartiles of LTßR (Figure 2) revealed a risk for incident GI cancer for the first quartile of $0.25 \%$, the second quartile of $0.82 \%$, the third quartile $1.16 \%$ and the fourth quartile $1.9 \%$ at 12 years $(\log$-rank $\mathrm{P}=0.011)$. The median followup (time interval from enrollment to the time of reported subsequent cancer diagnosis) for incident GI cancer was 5.9 $(2.8,8.9)$ years.

\section{Plasma LTBR and incident cancer type}

The major incident cancer types for the 178 participants were: breast 40 (22.5\%); prostate $30(16.9 \%)$ and GI 30 (16.9\%). After adjustments for age, race and sex, plasma LT $\beta R$ levels were not associated with the subsequently reported diagnosis of incident breast cancer (HR 1.69, 95\% CI, 0.70-4.13) or incident prostate cancer (HR 0.34, 95\% CI, 0.1-1.37) as depicted in Table 3. However, elevated levels of plasma LT $\beta R$ were positively associated with incident GI cancer, even after adjustment for age, race, and sex (HR 2.64, 95\% CI, 1.23-5.68, $\mathrm{P}=0.013$, Table 3).

\section{Plasma LTBR and anatomical sites of incident GI cancer}

The majority of the 30 incident GI cancers, were colon $(30 \%)$, rectal $(20 \%)$, pancreatic $(17 \%)$ and liver $(10 \%)$, as shown in Table S4. The highest mean LTßR level was liver: $2.10 \pm 0.61 \mathrm{ng} / \mathrm{mL}$ and the lowest mean LT $\beta \mathrm{R}$ level was pancreatic: $1.10 \pm 0.91 \mathrm{ng} / \mathrm{mL}$. Statistical analyses for the association of plasma LT $\beta \mathrm{R}$ and anatomical sites of incident GI cancer were limited by the low number of cases within each anatomical site as shown in Table S4. 
Table 3 Association of plasma lymphotoxin- $\beta$ receptor with specific incident cancer type adjusted for age, race, and sex

\begin{tabular}{lccc}
\hline & Breast cancer & Prostate & Gastrointestinal \\
\hline Lymphotoxin- $\beta$ receptor & $1.69(0.70-4.13) ; 0.25$ & $0.37(0.10-1.37) ; 0.14$ & $2.64(1.23-5.68) ; 0.013$ \\
\hline
\end{tabular}

Data reported as HR (95\% Cl); P value.

\section{Discussion}

The present longitudinal multiethnic population-based cohort study demonstrates that elevated plasma levels of the immune or inflammatory protein, LT $\beta$ R are associated with the subsequent development of incident GI cancer after adjustment for age, sex and race. To our knowledge, this is the first longitudinal human study to demonstrate that the antecedent elevated plasma LT $\beta$ R levels predate the subsequent diagnosis of incident GI cancer.

In the present study, similar findings that elevated plasma LT $\beta \mathrm{R}$ is associated with the subsequent development of incident prostate and breast cancers was not observed since the HR for the association of incident prostate cancer and LT $\beta R$ was 0.37 (Table 3), although it was not statistically significant. This is consistent with findings that LT $\beta R$ can exert an antitumor effect $(16,26)$. This opposite effect of LT $\beta R$ may account for why, in analysis of all incident cancers (which includes an admixture of prostate and GI cancers), a significant relationship was not observed, but a significant relationship was observed in an analysis restricted to incident GI cancer. In addition, these understanding underscore the need to perform separate analyses based on type of incident cancer (GI versus prostate). The relevance may also apply to the subtyping of incident GI cancers by anatomical sites; the mean plasma LT $\beta R$ levels for liver was higher when compared to pancreatic cancer. The apparent difference is supported by studies which clearly demonstrated increased expression of LT $\beta$ R in hepatocellular carcinoma, whereas comparable studies are not available for pancreatic cancer (17). Hence, a stronger relationship may potentially be demonstrated if the analyses were restricted to only hepatocellular carcinoma but did not include pancreatic cancer.

This differential effect based on type of cancer (GI versus prostate or breast) may reflect the critical role of LT $\beta R$ signaling in the immune and inflammatory responses of the GI system. LT $\beta R$ has been demonstrated in mice models to be essential for organogenesis in secondary lymphoid organs of the GI system such as Peyer's patches $(27,28)$. This was demonstrated in the knockout mouse model for the LT $\beta R$ gene when the mice failed to develop Peyer's patches $(28,29)$.
Moreover, the inflammatory GI responses to viral hepatitis, Helicobacter pylori and colitis are mediated by LT $\beta$ R $(17,30,31)$. Intriguingly, each of these inflammatory conditions with the liver, stomach and colon in humans is associated with an increased risk of subsequently developing a GI cancer in these organs.

The relevance of LT $\beta$ R to GI cancers is supported by studies that demonstrate LT $\beta$ R participates in the immune system development, combined with the findings of increased incidences of GI malignancies in patients with common variable immunodeficiency (26,27,32-34). In attempt to better understand the genetic basis for colorectal cancer, a GI cancer, Zhang et al. mapped 2 genetic loci to genes TCF7L2 and TGFB1 which are associated with $W n t / \beta$-catenin pathway $(35,36)$. In another pathway, Scarzello and colleagues demonstrated LT $\beta$ R signaling of AKT $/ \beta$-catenin-initiated tumors induced robust increase in proliferation and progression of hepatic tumors (37). To identify specific proteins that may participate in the pathways of tumorigenesis for GI cancers, Saha and colleagues identified high gene expression of TTYH3, an ionic channel membrane protein, that was associated with poorer clinical outcomes for gastric cancer and could have utility as a biomarker. LT $\beta$ R, a membrane protein, may also function in a similar manner to TTYH3 (38). These proteins may encourage future research in elucidating the pathogenetic mechanisms of tumorigenesis for GI cancers (38).

The association of LT $\beta \mathrm{R}$ with cancer, is also provided by experimental studies $(15,16)$. Unlike the present epidemiological study, experimental studies have examined LT $\beta R$ in the cell membrane but not plasma or soluble LT $\beta R$. It is not clear that the plasma LT $\beta R$ concentration mirrors the activity of LT $\beta R$ in the cell membrane. Several potential mechanisms may explain the finding of elevated levels of soluble or plasma LT $\beta$ R that positively affect tumor development. High levels of measurable soluble LT $\beta \mathrm{R}$ in the circulation may be the result of receptor proteolytic cleavage and shedding of cell-surface receptors, which is a common phenomenon observed with other cytokine receptors such as TNFR1 and IL6R $(39,40)$. It 
has been reported that sheddases, proteases that cleave the extracellular domains of receptors from the cell surface, are frequently overexpressed in cancer (39). Additionally, the cleaved extracellular domain of LT $\beta$ R can function as a decoy by binding to either LT $\alpha \beta_{2}$ or LIGHT and further reduce LT $\beta R$ mediated action.

Our findings are similar to other epidemiological studies, the Nurses' Health Study and the Tromsø Study which found that elevated plasma soluble tumor necrosis factor receptor 2 (sTNFR-2) and serum osteoprotegerin (OPG) levels were associated with the subsequent diagnosis of incident colorectal cancer and GI cancer, respectively $(7,8)$. The Tromsø study found that elevated serum levels of OPG prior to the diagnosis of incident cancer were associated with an increased risk of incident GI cancer and mortality, but not with incident breast or prostate cancers (7). The present study also congruently found that elevated levels of soluble plasma LT $\beta$ R predate the diagnosis of incident GI cancer but not breast or prostate cancers. OPG, TNFR-2 and LT $\beta$ R are inflammatory proteins and $\mathrm{OPG}$ is known to function as a decoy for tumor necrosis factor receptor superfamily member 11B (TNFRSF11B). Soluble LT $\beta$ R and sTNFR-2 potentially could function as decoys for tumor necrosis factor receptor superfamily member 3 (TNFRSF3) and TNFR-2, respectively. The similar findings from these three separate prospective epidemiological studies independently corroborate the individual results of each study. The findings from the present study are reinforced with the understanding that the DHS was not only a racially diverse study population but by study design there was an intentional oversampling of nonHispanic African American participants which was greater than $50 \%$ of the study population. This racial distribution was not a study design feature for either the Nurses' Health Study and the Tromsø Study.

The intriguing findings that the antecedent elevation of the plasma immune or inflammatory protein LT $\beta R$ at a median of 5.9 years predated the subsequent clinical detection or diagnosis of GI cancer suggests that LT $\beta$ R has an early role in the development and pathogenesis. Early elevations of this immune or inflammatory biomarker may correlate with the beginnings of tumorigenesis or the tumor microenvironment. The immune system is an important component in the tumor microenvironment and LT $\beta \mathrm{R}$ and its cognate ligands are immune protein mediators that shape the tumor microenvironment (10). Thus, elevated plasma LT $\beta R$ levels may be early in vivo immune biomarkers for the tumor microenvironment and the subsequent diagnosis of incident GI cancer.

Strengths of this study include the population-based design, large number of participants, racial heterogeneity with the intentional oversampling of African Americans, long-term follow-up and the simultaneous measurement of LT $\beta R$ in this cohort. Limitations include the observational design which precludes the ability to establish causality as well as the dynamic relationship of how these levels change over time. Due to the retrospective analysis of this cohort population, with limited impact on exposure and outcome assessment, this analysis and interpretation of data was hypothesis-generating, which needs to be confirmed in a prospective fashion. DHS participants who were diagnosed with cancer outside the state of Texas would not have been captured and reduced our sample size. Also, limited numbers of overall cancers and GI cancers $(\mathrm{N}=30)$ and should be combined with future studies to confirm the findings.

In summary, elevated plasma LT $\beta \mathrm{R}$ is associated with the subsequent diagnosis of incident GI cancer at a median interval of 5.9 years. The antecedent elevated LT $\beta$ R levels years prior to the development of incident GI cancers, combined with pre-clinical studies by other investigators suggest a role for LT $\beta R$ in the pathogenesis of GI cancer. These findings may provide insight into the tumor microenvironment and be potentially useful for risk stratification of GI cancer by utilizing circulating inflammatory biomarkers. Further studies are necessary to elucidate the mechanisms of tumorigenesis in GI cancer.

\section{Acknowledgments}

Cancer data provided by the TCR, Cancer Epidemiology and Surveillance Branch, Texas Department of State Health Services, 1100 West 49th Street, Austin, TX 78756. We acknowledge the DHS participants who unselfishly volunteered for this study. We would also like to thank Dr. Ezra Burstein, Dr. John Abrams and Dr. Yujin Hoshida for their contributions to the manuscript.

Funding: The Dallas Heart Study was funded by the Donald W. Reynolds Foundation and was partially supported by the National Center for Advancing Translational Sciences of the National Institutes of Health under award number UL1TR001105.

\section{Footnote}

Conflicts of Interest: The authors have no conflicts of interest 
to declare.

Ethical Statement: The authors are accountable for all aspects of the work in ensuring that questions related to the accuracy or integrity of any part of the work are appropriately investigated and resolved.

\section{References}

1. Hanahan D, Weinberg RA. Hallmarks of cancer: the next generation. Cell 2011;144:646-74.

2. Coussens LM, Werb Z. Inflammation and cancer. Nature 2002;420:860-7.

3. van der Woude CJ, Kleibeuker JH, Jansen PLM, et al. Chronic inflammation, apoptosis and (pre-)malignant lesions in the gastro-intestinal tract. Apoptosis 2004;9:123-30.

4. Crusz SM, Balkwill FR. Inflammation and cancer: advances and new agents Nat Rev Clin Oncol 2015;12:584-96.

5. Mehta RS, Song M, Bezawada N, et al. A prospective study of macrophage inhibitory cytokine-1 (MIC-1/ GDF15) and risk of colorectal cancer. J Natl Cancer Inst 2014;106:dju016.

6. Epplein M, Xiang YB, Cai Q, et al. Circulating cytokines and gastric cancer risk. Cancer Causes Control 2013;24:2245-50.

7. Vik A, Brodin EE, Mathiesen EB, et al. Serum osteoprotegerin and future risk of cancer and cancerrelated mortality in the general population: the Tromsø study. Eur J Epidemiol 2015;30:219-30.

8. Chan AT, Ogino S, Giovannucci EL, et al. Inflammatory markers are associated with risk of colorectal cancer and chemopreventive response to anti-inflammatory drugs. Gastroenterology 2011;140:799-808.

9. Song $\mathrm{M}, \mathrm{Wu} \mathrm{K}$, Ogino $\mathrm{S}$, et al. A prospective study of plasma inflammatory markers and risk of colorectal cancer in men. Br J Cancer 2013;108:1891-8.

10. Bjordahl RL, Steidl C, Gascoyne RD, et al. Lymphotoxin network pathways shape the tumor microenvironment. Curr Opin Immunol 2013;25:222-9.

11. Browning JL, Ngam-ek A, Lawton P, et al. Lymphotoxin beta, a novel member of the TNF family that forms a heteromeric complex with lymphotoxin on the cell surface. Cell 1993;72:847-56.

12. Crowe PD, VanArsdale TL, Walter BN, et al. A lymphotoxin-beta-specific receptor. Science 1994;264:707-10.

13. Force WR, Walter BN, Hession C, et al. Mouse lymphotoxin-beta receptor. Molecular genetics, ligand binding, and expression. J Immunol 1995;155:5280-8.

14. Remouchamps C, Boutaffala L, Ganeff C, et al. Biology and signal transduction pathways of the Lymphotoxin$\alpha \beta /$ LT $\beta R$ system. Cytokine Growth Factor Rev 2011;22:301-10.

15. Wolf MJ, Seleznik GM, Zeller N, et al. The unexpected role of lymphotoxin beta receptor signaling in carcinogenesis: from lymphoid tissue formation to liver and prostate cancer development. Oncogene 2010;29:5006-18.

16. Fernandes MT, Dejardin E, dos Santos NR. Contextdependent roles for lymphotoxin- $\beta$ receptor signaling in cancer development. Biochim Biophys Acta 2016;1865:204-19.

17. Haybaeck J, Zeller N, Wolf MJ, et al. A lymphotoxindriven pathway to hepatocellular carcinoma. Cancer Cell 2009;16:295-308.

18. Brown DA, Breit SN, Buring J, et al. Concentration in plasma of macrophage inhibitory cytokine- 1 and risk of cardiovascular events in women: a nested case-control study. Lancet 2002;359:2159-63.

19. Vik A, Mathiesen EB, Brox J, et al. Serum osteoprotegerin is a predictor for incident cardiovascular disease and mortality in a general population: the Tromsø Study. J Thromb Haemost 2011;9:638-44.

20. Shai I, Schulze MB, Manson JE, et al. A prospective study of soluble tumor necrosis factor-alpha receptor II (sTNFRII) and risk of coronary heart disease among women with type 2 diabetes. Diabetes Care 2005;28:1376-82.

21. Pai JK, Pischon T, Ma J, et al. Inflammatory markers and the risk of coronary heart disease in men and women. $\mathrm{N}$ Engl J Med. 2004 351:2599-610.

22. Owens AW, Matulevicius S, Rohatgi A, et al. Circulating lymphotoxin $\beta$ receptor and atherosclerosis: observations from the Dallas Heart Study. Atherosclerosis 2010;212:601-6.

23. Victor RG, Haley RW, Willett DL, et al. The Dallas Heart Study: a population-based probability sample for the multidisciplinary study of ethnic differences in cardiovascular health. Am J Cardiol 2004;93:1473-80.

24. Gupta A, Herman Y, Ayers C, et al. Plasma Leptin Levels and Risk of Incident Cancer: Results from the Dallas Heart Study. PLoS One 2016;11:e0162845.

25. Beg MS, Saleem S, Turer A, et al. A Prospective Analysis of Plasma Adiponectin and Risk of Incident Cancer: The Dallas Heart Study. J Natl Compr Canc Netw 2015;13:873-8. 
26. Hu X, Zimmerman MA, Bardhan K, et al. Lymphotoxin $\beta$ receptor mediates caspase-dependent tumor cell apoptosis in vitro and tumor suppression in vivo despite induction of NF- $\kappa$ B activation. Carcinogenesis 2013;34:1105-14.

27. Banks TA, Rouse BT, Kerley MK, et al. Lymphotoxinalpha-deficient mice. Effects on secondary lymphoid organ development and humoral immune responsiveness. J Immunol 1995;155:1685-93.

28. Fütterer A, Mink K, Luz A, et al. The lymphotoxin beta receptor controls organogenesis and affinity maturation in peripheral lymphoid tissues. Immunity 1998;9:59-70.

29. Spahn TW, Eugster HP, Fontana A, et al. Role of lymphotoxin in experimental models of infectious diseases: potential benefits and risks of a therapeutic inhibition of the lymphotoxin-beta receptor pathway. Infect Immun 2005;73:7077-88.

30. Feige $\mathrm{MH}$, Vieth $\mathrm{M}$, Sokolova $\mathrm{O}$, et al. Helicobacter pylori induces direct activation of the lymphotoxin beta receptor and non-canonical nuclear factor-kappa B signaling. Biochim Biophys Acta Mol Cell Res 2018;1865:545-50.

31. Stopfer P, Obermeier F, Dunger N, et al. Blocking lymphotoxin- $\beta$ receptor activation diminishes inflammation via reduced mucosal addressin cell adhesion molecule-1 (MAdCAM-1) expression and leucocyte margination in chronic DSS-induced colitis. Clin Exp Immunol 2004;136:21-9.

32. Mooster JL, Le Bras S, Massaad MJ, et al. Defective lymphoid organogenesis underlies the immune deficiency caused by a heterozygous S321 mutation in $1 \kappa \mathrm{B} \alpha$. J Exp Med 2015;212:185-202.

33. Pulvirenti F, Pecoraro A, Cinetto F, et al. Gastric Cancer Is the Leading Cause of Death in Italian Adult Patients With Common Variable Immunodeficiency. Front Immunol 2018;9:2546.

34. Leone P, Vacca A, Dammacco F, et al. Common variable immunodeficiency and gastric malignancies. Int J. Mol Sci 2018;19:451.

35. Zhang B, Jia WH, Matsuda K, et al. Large-scale genetic study in East Asians identifies six new loci associated with colorectal cancer risk. Nat Genet 2014;46:533-42.

36. Tang W, Dodge M, Gundapaneni D, et al. A genome-wide RNAi screen for Wnt/beta-catenin pathway components identifies unexpected roles for TCF transcription factors in cancer. Proc Natl Acad Sci USA 2008;105:9697-702.

37. Scarzello AJ, Jiang Q, Back T, et al. LT R signaling preferentially accelerates oncogenic AKT-initiated liver tumours. Gut 2016;65:1765-75.

38. Saha SK, Biswas PK, Gil M, et al. High Expression of TTYH3 is Related to Poor Clinical Outcomes in Human Gastric Cancer. J Clin Med 2019;8:1762.

39. Miller MA, Sullivan RJ, Lauffenburger DA. Molecular Pathways: Receptor Ectodomain Shedding in Treatment, Resistance, and Monitoring of Cancer. Clin Cancer Res 2017;23:623-9.

40. Levine SJ. Molecular mechanisms of soluble cytokine receptor generation. J Biol Chem 2008;283:14177-81.
Cite this article as: Bergstrom CP, Beg MS, Ayers C, Gupta A, Neeland IJ. The association of lymphotoxin-beta receptor with the subsequent diagnosis of incident gastrointestinal cancer: results from the Dallas Heart Study. J Gastrointest Oncol 2020;11(1):36-44. doi: 10.21037/jgo.2020.01.04 